OPEN ACCESS

Edited by: Kai He,

Lanzhou University, China

Reviewed by:

Frantisek Baluska,

University of Bonn, Germany

Norbert Rolland,

Centre National de la Recherche

Scientifique (CNRS), France

*Correspondence:

Jian-Zhong Liu

jzliu@zjnu.cn

${ }^{\dagger}$ These authors have contributed equally to this work.

Specialty section:

This article was submitted to

Plant Traffic and Transport,

a section of the journal

Frontiers in Plant Science

Received: 06 July 2017 Accepted: 03 November 2017

Published: 23 November 2017

Citation:

Ni M, Zhang L, Shi Y-F, Wang C, LU Y, Pan J and Liu J-Z (2017) Excessive

Cellular S-nitrosothiol Impairs Endocytosis of Auxin Efflux Transporter PIN2.

Front. Plant Sci. 8:1988. doi: 10.3389/fp/s.2017.01988

\section{Excessive Cellular S-nitrosothiol Impairs Endocytosis of Auxin Efflux Transporter PIN2}

\author{
Min $\mathrm{Ni}^{1+}$, Lei Zhang ${ }^{1 \dagger}$, Ya-Fei Shi ${ }^{1 \dagger}$, Chao Wang ${ }^{2 \dagger}$, Yiran $\mathrm{Lu}^{1}$, Jianwei Pan ${ }^{1,2}$ and \\ Jian-Zhong Liu ${ }^{1 *}$ \\ ${ }^{1}$ College of Chemistry and Life Sciences, Zhejiang Normal University, Jinhua, China, ${ }^{2}$ Ministry of Education Key Laboratory of \\ Cell Activities and Stress Adaptations, School of Life Sciences, Lanzhou University, Lanzhou, China
}

S-nitrosoglutathione reductase (GSNOR1) is the key enzyme that regulates cellular levels of S-nitrosylation across kingdoms. We have previously reported that loss of GSNOR1 resulted in impaired auxin signaling and compromised auxin transport in Arabidopsis, leading to the auxin-related morphological phenotypes. However, the molecular mechanism underpinning the compromised auxin transport in gsnor1-3 mutant is still unknown. Endocytosis of plasma-membrane (PM)-localized efflux PIN proteins play critical roles in auxin transport. Therefore, we investigate whether loss of GSNOR1 function has any effects on the endocytosis of PIN-FORMED (PIN) proteins. It was found that the endocytosis of either the endogenous PIN2 or the transgenically expressed PIN2-GFP was compromised in the root cells of gsnor1-3 seedlings relative to Col-0. The internalization of PM-associated PIN2 or PIN2-GFP into Brefeldin A (BFA) bodies was significantly reduced in gsnor1-3 upon BFA treatment in a manner independent of de novo protein synthesis. In addition, the exogenously applied GSNO not only compromised the endocytosis of PIN2-GFP but also inhibited the root elongation in a concentration-dependent manner. Taken together, our results indicate that, besides the reduced PIN2 level, one or more compromised components in the endocytosis pathway could account for the reduced endocytosis of PIN2 in gsnor1-3.

Keywords: endocytosis, nitric oxide, PIN-FORMED (PIN) proteins, polar auxin transport, S-nitrosoglutathione reductase

\section{INTRODUCTION}

Nitric oxide (NO) is a reactive free radical gaseous molecule that is involved in battery of biological processes both in animals and plants (Wendehenne et al., 2014). In plants, NO participates in biological processes such as stomatal closure, cell death and disease resistance, abiotic stress, flowering, and many other processes (Durner et al., 1998; Klessig et al., 2000; Neill et al., 2002; Lamattina et al., 2003; He et al., 2004, 2012; Wendehenne et al., 2004, 2014; Zeidler et al., 2004; Lee et al., 2008; Xuan et al., 2010; Fan and Liu, 2012; Lin et al., 2012; Ye et al., 2012; Mur et al., 2013).

$S$-nitrosylation, adding NO moiety to a protein, is a novel mechanisms by which NO regulates protein functions (Hess and Stamler, 2012; Wendehenne et al., 2014). This non-enzymatic reversible protein modification is analogous to protein phosphorylation (Stamler et al., 1992; Hess et al., 2005). Many proteins have been identified as targets of $S$-nitrosylation and their functions 
are regulated by this modification (Lindermayr et al., 2005; Forrester et al., 2009; Hess and Stamler, 2012; Yang et al., 2015). In plants, the target cysteine residues of some $S$-nitrosylated proteins have been identified and the functional importance of this modification is unraveled (Lindermayr et al., 2006, 2010; Belenghi et al., 2007; Romero-Puertas et al., 2007; Serpa et al., 2007; Tada et al., 2008; Chen et al., 2009; Wang et al., 2009, 2015; Yun et al., 2011; Astier et al., 2012; Feng et al., 2013; Yang et al., 2015; Hu et al., 2017; Liu et al., 2017).

The level of cellular protein $S$-nitrosylation is dynamic and governed by NO levels and de-nitrosylation catalyzed by $S$ nitrosoglutathione reductase (GSNOR) (Liu et al., 2001; Feechan et al., 2005) and thioredoxin (Tada et al., 2008; Benhar et al., 2009; Sengupta and Holmgren, 2012). GSNOR is the key enzyme controlling $S$-nitrosoglutathione (GSNO) levels by reducing GSNO to oxidized GSH and $\mathrm{NH}_{3}$ and thus indirectly controls the cellular levels of $S$-nitrosylated proteins (Liu et al., 2001, 2004; Feechan et al., 2005).

Auxin is one of mostly studied plant hormone that plays diverse roles in development (Teale et al., 2006). Auxin gradients, which are created and maintained by groups of transporters localized on plasma membrane (PM) are critical to auxin functions in the regulation of stem cell differentiation, the initiation of lateral organs and gravitropic responses (Woodward and Bartel, 2005; Leyser, 2006; Petrásek and Friml, 2009). One of the most important transporters is the PIN-FORMED (PIN) family of auxin efflux proteins (Chen et al., 1998; Galweiler et al., 1998; Muller et al., 1998; Geldner et al., 2001; Blilou et al., 2005; Wisniewska et al., 2006; Pan et al., 2009).

Clathrin-mediated endocytosis (CME) is an evolutionally conserved pathway that plays a critical role in determining protein abundance at the PM and/or the trans-Golgi network (TGN) during signaling transductions and retargeting/degradation of proteins at PM (Chen et al., 2011; McMahon and Boucrot, 2011; Wang et al., 2013). CME is the predominant pathway for the internalization of numerous membrane-localized proteins including PINs (Paciorek et al., 2005). By inhibiting the endocytosis of PIN, auxin increases levels of various PINs at the PM (Paciorek et al., 2005). As a result, auxin promotes its own efflux by vesicle-traffickingdependent mechanism (Paciorek et al., 2005). In addition to CME, a BFA-insensitive and clathrin-independent endocytic route has also been reported for PM resident proteins (Beck et al., 2012).

We have previously reported that loss of GSNOR1 function in Arabidopsis impairs both auxin signaling and polar auxin transport and thereby the gsnor1-3 mutant displays multiple auxin-related morphological defects including short and highly branched statures, short primary roots, and lack of lateral roots. The compromised polar auxin transport in gsnor 1-3 is due to universally reduced levels of auxin efflux transporters PIN proteins at the plasma membrane (PM) (Shi et al.,

Abbreviations: 2,4-D, 2,4-dichloropheenoxyacetic; CME, Clathrin-mediated endocytosis; CHX, cycloheximide; NO, Nitric oxide; GSNO, S-nitrosoglutathione; GSNOR1, S-nitrosoglutathione reductase 1; SNO, S-nitrosothiol; TGN, TransGolgi network.
2015). However, whether loss of GSNOR1 inhibits polar auxin transport exclusively through reducing the abundance of PINs at PM or additional mechanisms are also involved, are largely unknown. Here, we showed that loss of GSNOR1 inhibited the internalization of either the transgenically expressed PIN2-GFP or the endogenous PIN2 independent of de novo protein synthesis and this inhibition could be recapitulated by exogenously applied GSNO. Furthermore, similar to loss of GSNOR1, exogenously applied GSNO inhibited the root elongation in a concentration dependent manner. Together, our results reveal an additional layer of complex roles of $\mathrm{NO}$ in regulating plant growth and development through modulating internalization of auxin efflux transporter.

\section{RESULTS}

\section{Loss of GSNOR1 Results in Reduced Internalization of Transgenically Expressed PIN2-GFP}

To examine the effect of NO signaling on the internalization of the PM-associated PIN proteins, we used Brefeldin A (BFA; $50 \mu \mathrm{M}$ ), a vesicle trafficking inhibitor (Geldner et al., 2001), to visualize the PIN2-GFP internalization in the wild-type and gsnor1-3 seedlings that express the PIN2-GFP driven by its native promoter (ProPIN2:PIN2-GFP). Consistent with our previous report (Shi et al., 2015), the intensity of the PIN2-GFP fluorescence at the PM was significantly reduced in gsnor1-3 mutants relative to the wild-type cells under mock conditions (compare Figures 1A,D; and Figure 1G). As expected, both the numbers and the relative intensities of the PIN2-GFPlabeled BFA bodies were also significantly reduced in the gsnor 13 compared to the wild-type cells (compare white arrowpointed BFA bodies in Figures 1B,E; and see statistical data in Figures $1 \mathrm{H}, \mathbf{I}$ ), indicating that the internalization of the PIN2GFP was reduced in the gsnor1-3 mutants. To dissect whether the reduced numbers and intensities of the BFA-induced PIN2GFP fluorescent bodies in gsnor1-3 is exclusively resulted from the reduced levels of PIN2-GFP at the PM, we further analyzed the ratios of GFP signals in BFA bodies to those at the PM both in Col-0 and gsnor1-3, respectively, after BFA treatment. As shown in Figure 1J, the GFP signal ratio of BFA bodies/PM was significantly lower in gsnor 1-3 mutants than in the wild-type cells, indicating that, besides the reduced level of the PIN2-GFP at the PM, PIN2-GFP internalization itself is also compromised in the gsnor1-3 mutant seedlings.

Auxin inhibits internalization of PM proteins (Paciorek et al., 2005). To address whether auxin inhibitory effect on PIN2 endocytosis is altered in the gsnorl-3 mutants, we treated the transgenic seedlings expressing the PIN2-GFP both in the Col-0 and the gsnor1-3 firstly with $10 \mu \mathrm{M} 2,4-\mathrm{D}$ for $30 \mathrm{~min}$ and followed by treatment with $10 \mu \mathrm{M} 2,4$-D plus $50 \mu \mathrm{M}$ BFA for additional $60 \mathrm{~min}$ as described (Wang et al., 2013). As shown in Figure 1, 2,4-D similarly blocked the PIN2-GFP internalization both in the wild-type and the mutant cells (compare 1C and 1F), indicating that auxin inhibition of PIN2 internalization is not significantly impaired in the gsnorl-3 mutant. 


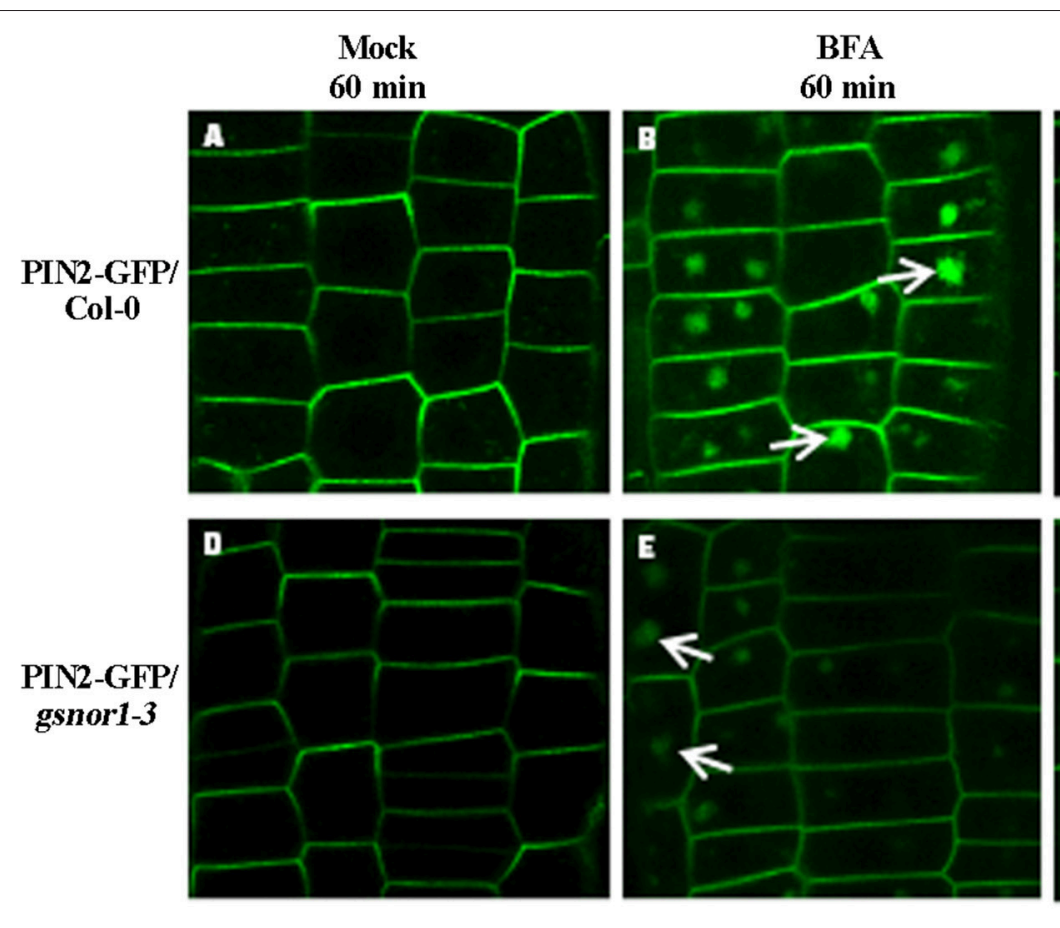

G

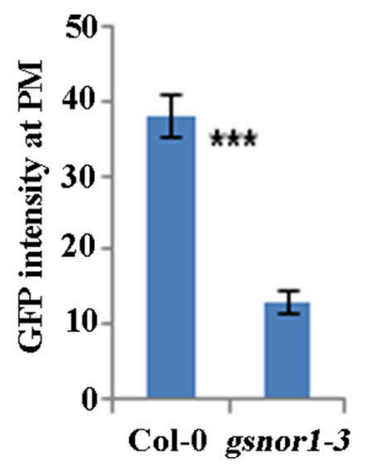

H

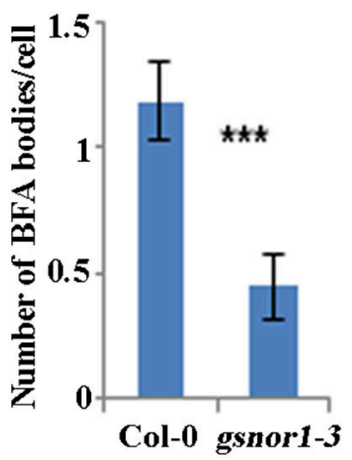

I

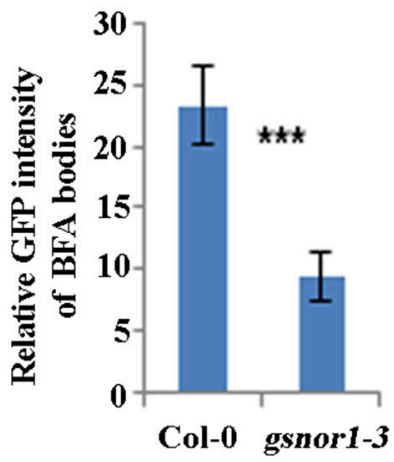

2,4-D/2,4-D+BFA $30 / 60 \mathrm{~min}$
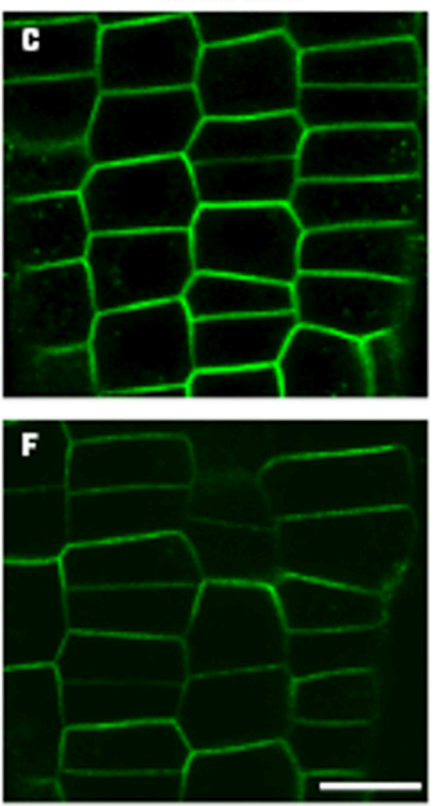

FIGURE 1 | The internalization and not the auxin inhibition of internalization of the transgenically expressed PIN2-GFP is impaired in gsnor1-3 mutant. Six-day-old ProPIN2:PIN2-GFP-expressing transgenic seedlings either in Col-0 or in gsnor1-3 background were treated with Mock (A,D), $50 \mu \mathrm{M}$ BFA for 60 min (B,E), and with $10 \mu \mathrm{M} 2$, 4-D for $30 \mathrm{~min}$, followed by treatment with $50 \mu \mathrm{M}$ BFA+ $10 \mu \mathrm{M}$ 2, 4-D for additional 60 min (C,F). Images were captured by confocal laser scanning microscopy (CLSM, Leica TCS SP5 AOBS). The numbers of BFA bodies (see white arrows) were counted and the fluorescence intensities both at the BFA bodies and at the PM were measured, respectively, using Image $\mathrm{J}$ (http://rsb.info.nih.gov/ij) and the statistical data were summarized (G-J). (G) GFP intensity at the PM; (H) Number of BFA bodies per cell; (I) Relative GFP intensities of the BFA bodies; (J) The GFP intensity ratios of the BFA bodies/PM. Bar = 50 $\mu \mathrm{m}$. ${ }^{\star \star \star}$ Indicates significant differences between Col-0 and gsnor $1-3$ by Student's $t$-test at 0.001 level.

\section{Loss of GSNOR1 Results in Reduced Internalization of the Endogenous PIN2}

Next, to test whether the internalization of endogenous PIN2 is similarly impaired as PIN2-GFP in the gsnor1-3 mutant, we performed immunofluorescence microscopy analysis using affinity-purified anti-PIN2-specific antibodies (Wang et al., 2013). Similar to the PIN2-GFP shown in Figure 1, the level of the PM-localized endogenous PIN2 was significantly reduced in the gsnor1-3 cells compared to the wild-type cells under the mock conditions (compare Figures 2A,D and Figure 2G).
Similarly, the numbers and the relative intensities of PIN2labeled BFA bodies (compare the white arrow-pointed BFA bodies in Figures 2B,E; also see statistical data in Figures 2H,I) and the fluorescence intensity ratio of BFA bodies/PM of the endogenous PIN2 (Figure 2J) were all significantly decreased in the gsnor1-3 mutant compared to the wild-type cells. Again, the inhibition of the PIN2 internalization in the presence of 2,4-D was not significantly altered in the gsnor1-3 mutant compared to the Col-0 cells (Figures 2C,F, no visible BFA bodies). These results confirmed the conclusions drawn from 
the studies using the ProPIN2:PIN2-GFP-expressing transgenic seedlings (Figure 1), suggesting that the transgenic expressed PIN2-GFP driven by its own promoter can recapitulate the endogenous PIN2.

\section{Loss of GSNOR1 Results in the Reduced Endocytosis of PIN2-GFP in the Absence of de novo Protein Synthesis}

To accurately assess the effect of loss of GSNOR1 on the internalization of PM-localized PIN2-GFP, the interference of the newly synthesized PIN2-GFP on the level of PM-localized PIN2GFP must be excluded. To do so, we firstly treated the 6-dayold seedlings with cycloheximide (CHX; $50 \mu \mathrm{M})$, an inhibitor of de novo protein synthesis, and followed by washout with CHX plus BFA. As shown in Figure 3, the GFP intensity on the $\mathrm{PM}$, the number of BFA bodies per cell and the relative GFP intensity of BFA bodies were all significantly reduced in gsnor 13 mutant seedlings relative to the wild-type cells after treatment with CHX for $30 \mathrm{~min}$ and followed by washout with $\mathrm{CHX}$ and BFA for additional $15 \mathrm{~min}$ or $60 \mathrm{~min}$ (Compare Figure $3 \mathrm{~A}$ and Figure 3D; Figure 3B and Figure 3E; Figure 3C and Figure 3F; and see statistical data shown in Figures 3G-I). Consistent with the results obtained without CHX treatment (Figure 1I), the GFP intensity ratio of BFA bodies/PM was significantly reduced in the gsnor 1-3 mutant cells relative to the wild-type cells after CHX and BFA co-treatment (Figure 3J). Accordingly, the relative level of the PM-localized GFP signal was higher in the gsnor13 than in Col-0 after CHX and BFA co-treatment (Figure 3K). These results again indicate that the PIN2-GFP internalization is impaired in the gsnor 1-3 even without de novo protein synthesis.

\section{The Exogenously Applied GSNO Recapitulates the Loss of GSNOR1 in Inhibiting PIN2-GFP Internalization}

We reasoned that if the impaired internalization of the PIN2-GFP or PIN2 observed in the gsnor1-3 (Figures 1-3) is indeed resulted from over-accumulation of cellular SNO, the exogenously applied GSNO should have a similar inhibitory effect on PIN2 internalization. To test this hypothesis, we tested the effect of exogenous GSNO treatment on the PIN2-GFP internalization in the wild-type cells. Consistent with the results obtained using the gsnor1-3 mutant (Figures 1, 2), exogenously applied GSNO not only reduced the level of the PM-localized PIN2-GFP (Figures 4A-C) but also inhibited the PIN2-GFP internalization in the presence of BFA in a concentration-dependent manner (Figures 4E-G, and statistical data in Figures 4I,J). These results suggest that the impaired polar auxin transport observed in the gsnor1-3 mutant seedlings (Shi et al., 2015) could be resulted at least partially from the reduced internalization of PIN2 (Figures 4D,H-J).

\section{Exogenous GSNO Inhibits Root Elongation in a Concentration Dependent Manner}

Our previous studies have shown that loss of GSNOR1 impairs auxin signaling and transport and the gsnorl-3 mutant plants display a wide range of auxin-related morphological defects including a short root phenotype (Shi et al., 2015). We postulated that if the short root phenotype observed in the gsnorl-3 mutant seedlings is indeed a consequence of cellular SNO overaccumulation, the exogenously applied GSNO could mimic the effects of the loss-of-function mutant of GSNOR1. To test this postulation, we treated the Col-0 seedlings with different concentrations of GSNO and used the gsnor1-3 mutant seedlings as a control. As expected, the exogenously applied GSNO inhibited the root elongation of the wild type seedlings in a concentration-dependent manner (Figures 5A-C), confirming that the excessive cellular SNO is at least partially, if not fully, responsible for the short root phenotype of the gsnorl-3 mutant seedlings (Figures 5D,E, and Shi et al., 2015).

\section{DISCUSSION}

It is not an uncommon phenomenon that phytohormones play roles in regulating CME. It has been reported that strigolactones affect shoot branching by modulating the endocytosis of PIN1 (Shinohara et al., 2013), and salicylic acid (SA) represses endocytosis of different PM-associated proteins by blocking clathrin recruitment at the PM (Du et al., 2013). Auxin inhibits the CME of several PM-localized proteins, including several PIN proteins (Paciorek et al., 2005). As a result, auxin promotes its own efflux by inhibiting the internalization of PINs and increases various PIN levels at the PM (Paciorek et al., 2005). In this report, we provided evidence that $\mathrm{NO}$, the other phytohormone, also play a role in the regulation of PIN2 endocytosis.

Our previous results have shown that even though the transcript level of PIN2-GFP was higher in the gsnor1-3 mutant than in the wild type Col-0, the intensity of PIN2-GFP at the PM was significantly reduced in the gsnor 1-3 mutant relative to the wild-type plants (Shi et al., 2015). Likely, the reduced accumulation of PIN2 at the PM in the gsnor1-3 could be partially resulted from compromised protein synthesis and/or stability and the reduced levels of the various PIN proteins in the gsnorl-3 could be the primary cause of the compromised polar auxin transport (Shi et al., 2015). However, our present data uncover that, in addition to the reduced level of PIN2 at the PM, the internalizations of PIN2 was also compromised in the gsnor1-3 mutant seedlings (Figures 1-3). Post-translational modifications play critical roles in regulating endocytosis. Both mono- and poly-ubiquitylation of single lysine is associated with cargo internalization and the intracellular sorting and targeting of PM proteins to the vacuole/lysosome rely on K63-linked ubiquitylation (Luschnig and Vert, 2014). PIN2 is modified by K63-linked poly-ubiquitin chains, which dependent on a class of ring-domain E3 ligase (RGLGs) (Yin et al., 2007; Leitner et al., 2012). Protein phosphorylation has been identified as a major determinant of PIN sorting and the sorting decision is dependent on the phosphorylation status of PINs and the activity of the serine/threonine protein kinase PINOID (PID) and its related proteins impact polar PIN distribution (Friml et al., 2004). As NO can also regulate protein functions by $S$-nitrosylation, it is highly possible that NO regulates internalization either directly by $S$ nitrosylating PINs or indirectly by $S$-nitrosylating the other key 


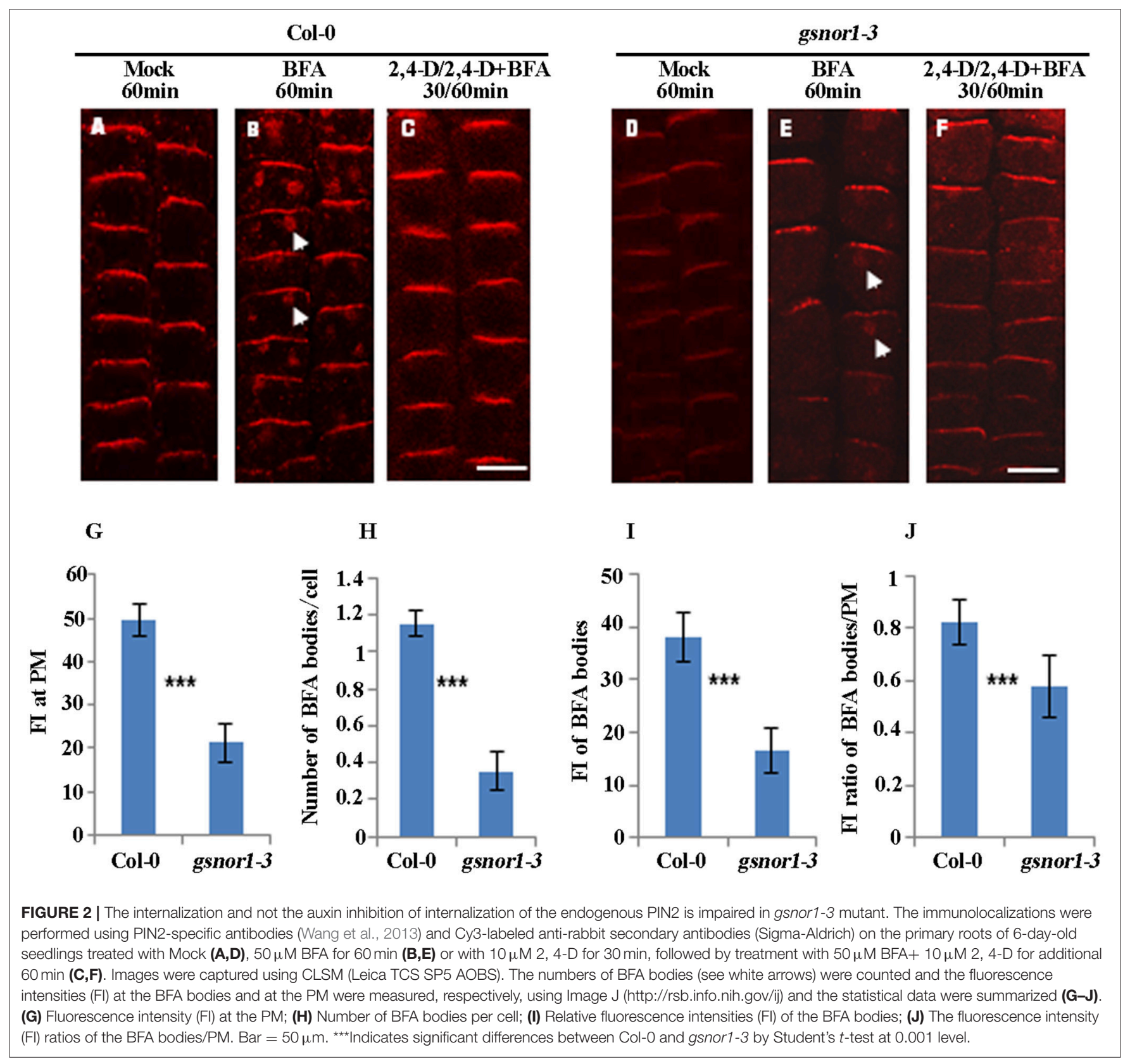

proteins in the endocytic pathways. This statement is supported by the facts that the activities of many PM-resident ion channels in animals, including $\mathrm{Na}^{+}$and $\mathrm{Ca}^{2+}$ channels, are regulated by S-nitrosylation (Xu et al., 1998; Renganathan et al., 2002) and Snitrosylation is invloved in modification of an outward-rectifying $\mathrm{K}^{+}$channel in Vicia faba (Sokolovski and Blatt, 2004).

Theoretically, the relatively enhanced level of PIN2-GFP at PM could relatively enhance the auxin polar transport (Paciorek et al., 2005). However, we observed a much reduced polar auxin transport in the gsnor 1-3 mutant primarily because of the universally reduced levels of PINs (Shi et al., 2015). One possibility is that the positive contribution of the relatively enhanced PM-localized PIN2, as a result of impaired internalizations, to polar auxin transport is masked by the inhibition resulted from the overall reduced level of PIN2 (Figures 1, 2; Shi et al., 2015). The other possibility is that, even though the relative level of PM-localized PIN2 is enhanced in the gsnor1-3 mutant relative to WT, the function of the PM-localized PIN2 is impaired under the excessive SNO condition, probably by $S$-nitrosylation. The functional endocytosis/exocytosis is required for replenishing the non-functional PM-localized PIN2 with newly synthesized functional PIN2 at PM. As a result, the reduced level of PIN2 and the compromised internalization of PIN2 (Figures 1-3) could contribute additively to the impaired polar auxin transport in the gsnor1-3 mutant and thus its auxinrelated morphological phenotypes. 


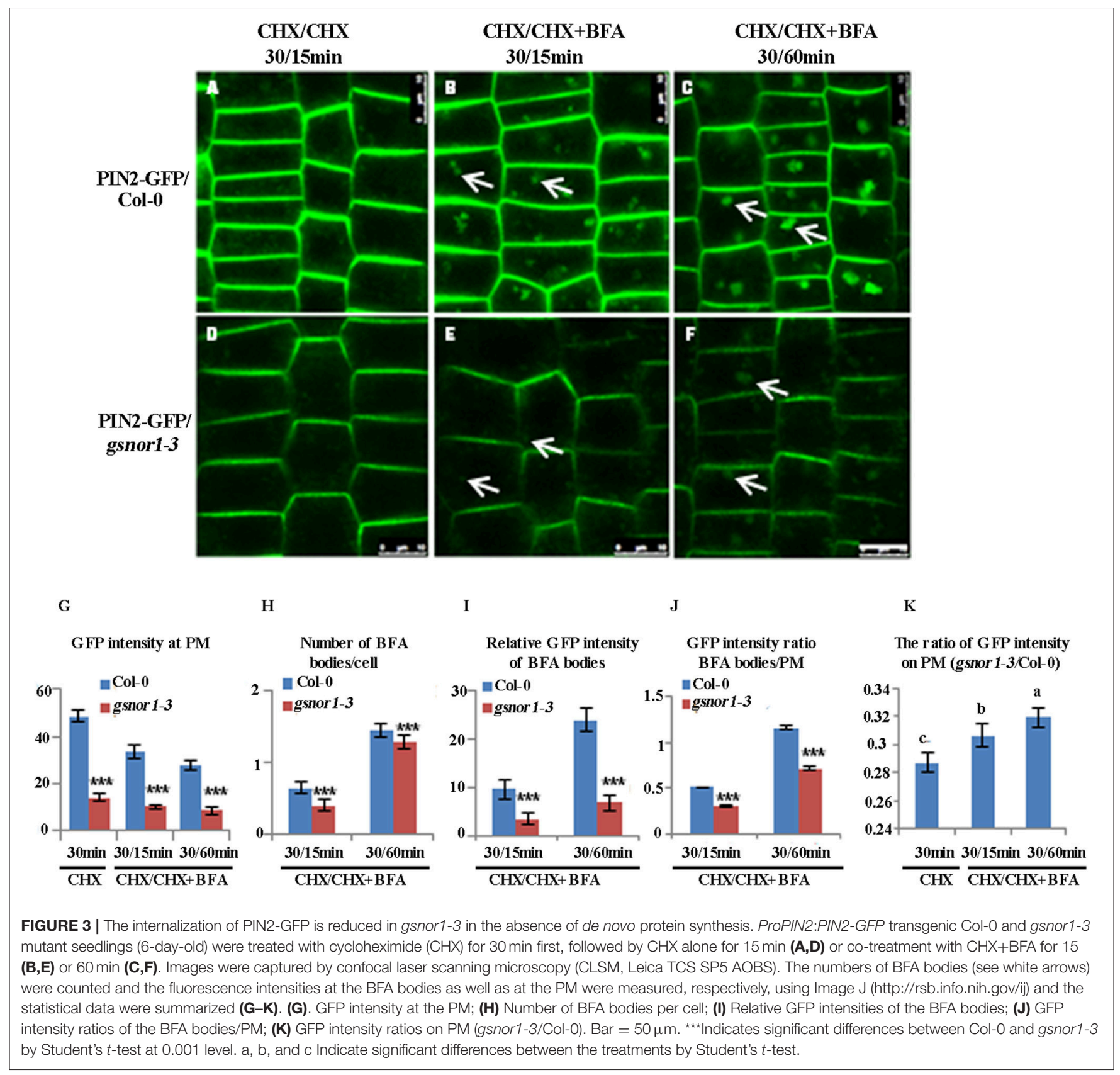

Even though the auxin signaling is significantly impaired in the gsnor1-3 (Shi et al., 2015), the auxin inhibition of either the transgenically expressed PIN2-GFP or the endogenous PIN2 internalization is not affected in the gsnor1-3 (Figures 1C,F, 2C,F), suggesting that auxin signaling does not play a critical role in the inhibition of PIN2 internalization. In agreement with this, it has been shown that differential auxin regulation of CLC and CHC membrane association is ABP1-dependent but TIR1/AFBindependent (Wang et al., 2013) and the role of ABP1 in auxin signaling is questionable (Gao et al., 2015; Liu, 2015). Given that the clathrin are required for the auxin inhibition of the PIN2GFP internalization (Wang et al., 2013) and the auxin inhibition of PIN2-GFP is not affected in gsnor1-3 mutant (Figures 1C,F, $2 \mathrm{C}, \mathrm{F}$ ), it is suggested that the reduced internalization of PIN2GFP or endogenous PIN2 (Figures 1, 2) may not be clathrindependent. In supporting this, it has been reported previously that the same PM protein could traffic through distinct endocytic routes (Beck et al., 2012). The activated FLS2 triggered by flg22 recognition at $\mathrm{PM}$ is targeted to the intracellular compartments for degradation via an ESCRT1-dependent, but BFA-insensitive route to prevent excessive and constitutive activation of defense signaling, whereas the non-activated or newly synthesized FLS2 is shuttled between PM and cytoplasm via a BFA-sensitive route (Robatzek et al., 2006; Beck et al., 2012; Spallek et al., 2013). 


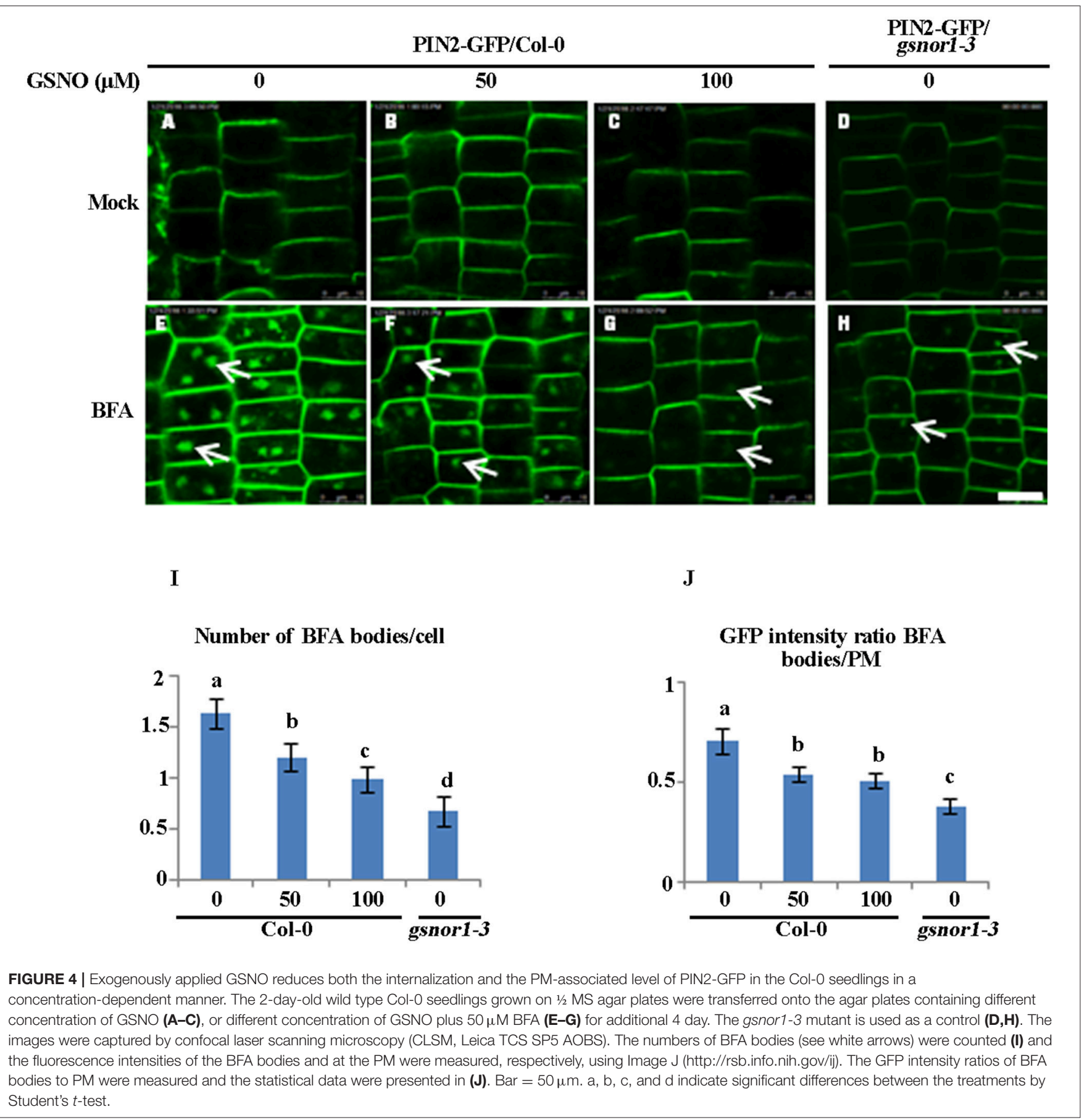

One may argue that the fluorescence intensity of some BFA bodies in gsnor1-3 mutant (Figures 1E, 2E) could be below the detection threshold under our Confocal settings, and therefore could have an impact on the fluorescence intensity ratio of BFA bodies/PM in the mutant. If that is really the case, the average fluorescence intensity of the BFA bodies within a cell or in a given captured image (5-20 root cells/image) of gsnor 1-3 root should be lower, and thus the fluorescence intensity ratio of BFA bodies/PM in the mutant should be lower than shown in Figures 1J, 2J. As a result, the detection thresholds issue would not change our final conclusion that the internalization of either the transgenically expressed PIN2-GFP or the endogenous PIN2 was compromised in the gsnor1-3 mutant.

In this report, we provided genetic and pharmaceutical evidence that the NO signaling plays an inhibitory role in PIN2 internalization. However, the molecular mechanism underlying the inhibition still remains unanswered. The questions like how NO signaling inhibits PIN2 internalization and what 


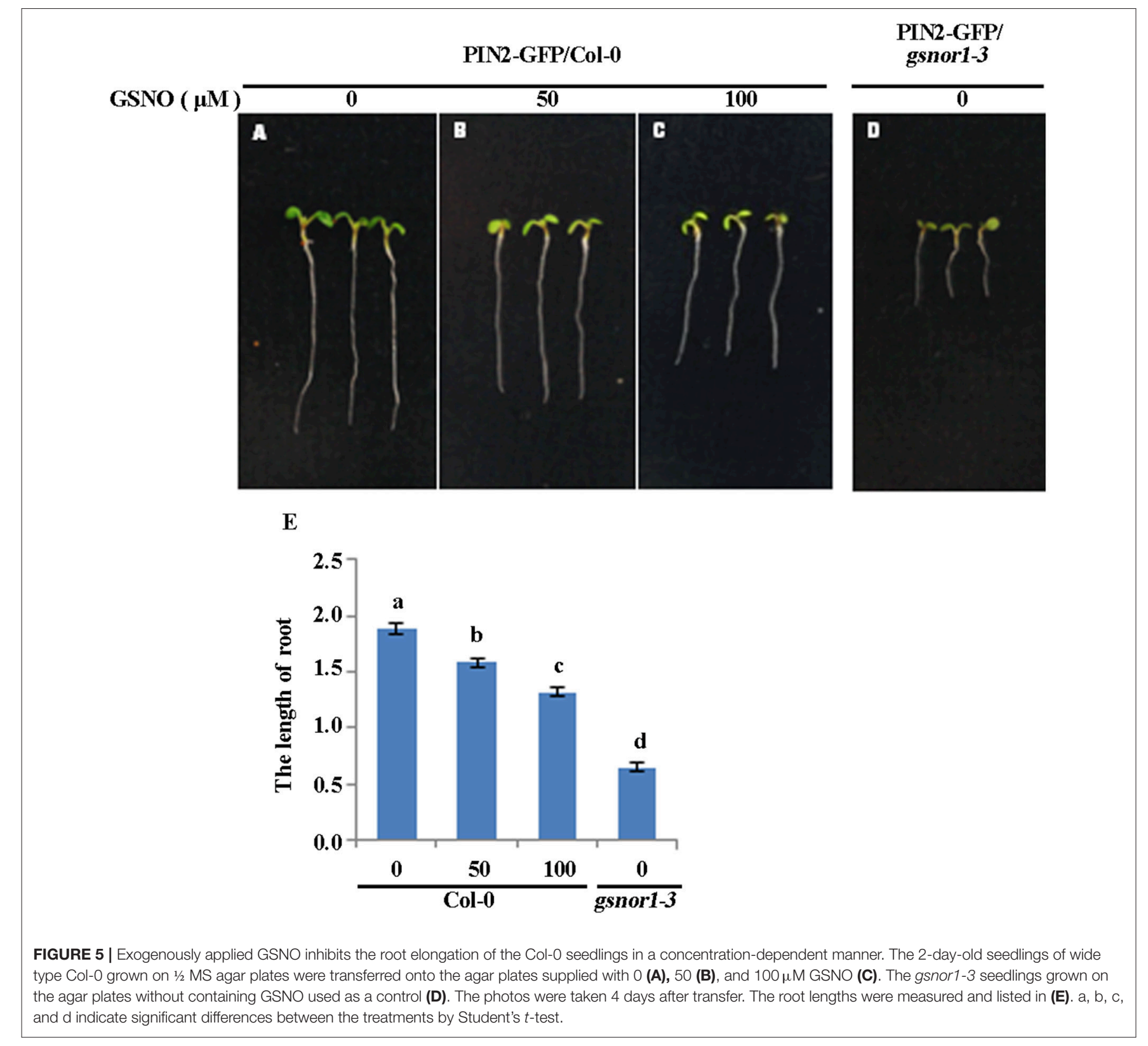

is the causal relationship between the NO-inhibited PIN2 internalization and polar auxin transport need to be addressed in the future. Nonetheless, our results reveal an additional layer of complex roles of NO in regulating plant growth and development through modulating internalization of auxin efflux transporter.

\section{MATERIALS AND METHODS}

\section{Arabidopsis Lines and Growth Conditions}

Arabidopsis Col-0 and gsnor1-3 (Feechan et al., 2005), the ProPIN2:PIN2-GFP transgenic line (Xu and Scheres, 2005) were used in this study. The ProPIN2:PIN2-GFP transgene was introgressed into the gsnor1-3 background by crossing. The homozygous gsnor1-3 line that expresses the ProPIN2:PIN2-GFP transgene was identified by the genomic PCR in combination of phenotype characterization among F3 population. These plants were grown under $16 \mathrm{~h}$-light $\left(22^{\circ} \mathrm{C}\right) / 8 \mathrm{~h}$-dark $\left(18^{\circ} \mathrm{C}\right)$. Seedlings were grown on $1 / 2 \mathrm{MS}$ medium containing $1.0 \%$ sucrose ( $\mathrm{pH}$ 5.7). Healthy six-day-old seedlings were used in this study.

\section{Chemical Solutions}

The stock solutions of CHX (50 mM, Sigma-Aldrich), BFA (50 mM; Invitrogen) and GSNO (100 mM, Sigma-Aldrich) were prepared in DMSO. 2,4-D (10 mM, Sigma-Aldrich) was firstly dissolved in $1 \mathrm{M} \mathrm{KOH}$ and then diluted with water as described (Wang et al., 2013). 


\section{Chemical Treatments}

All chemical pretreatments except GSNO were for $30 \mathrm{~min}$, and chemical treatment time is indicated in the text. For GSNO treatment, 2-day-old seedlings were transferred from GSNO-free 1/2 MS agar plates (containing 1.0\% sucrose, $\mathrm{pH}$ 5.7) into agar plates containing different concentrations of GSNO as indicated in the text for additional 3-4 days. All seedlings were grown on MS basal salts with minimal organics (Sigma Aldrich) supplemented with 1\% (w/v) Sucrose and 0.05\% (w/v) MES-KOH, pH 5.6 (0.5 3 x MS), liquid medium, except otherwise specified. The final $\mathrm{pH}$ of the medium was 5.6-5.7.

\section{Polyclonal Antibody and \\ Immunofluorescence Microscopic Analysis}

Polyclonal antibody, anti-AtPIN2, was raised in rabbits as described using a synthesized peptide (Wang et al., 2013) coupled with keyhole limpet hemocyanin containing an additional $\mathrm{N}$-terminal Cys (Huabio). Immunofluorescence microscopic analysis was performed as described (Wang et al., 2013). The primary PIN2 antibody was detected using Cy3-labeled anti-rabbit secondary antibodies (Sigma-Aldrich). Fluorescence images were captured using CLSM (Leica TCS SP5 AOBS). Excitation wavelengths for GFP and Cy3 were $488 \mathrm{~nm}$ (argon laser) and $561 \mathrm{~nm}$ (diode laser), respectively. The emission wavelengths were $496-532 \mathrm{~nm}$ for GFP and $550-570 \mathrm{~nm}$ for Cy3, respectively. For the purpose of direct comparisons of fluorescence intensities, laser, pinhole, and gain settings of the confocal microscope were set exactly identical when capturing the images from the seedlings of different genotypes or treatments. The intensities of fluorescence signals both at the PM and the cytoplasmic vesicles were quantified on the captured digital images using Image J (http://rsb.info.nih.gov/ ij) and the relative fluorescence intensities were presented as percentages of mock controls as described (Sauer et al., 2006; Robert et al., 2010). For the measurement of the fluorescence levels at the PM, optimal sections of the root cells were used for measurements. Using Image-J, PM regions of a captured images (over 20 cells for Figure 1; 5-10 cells for Figures 2-4) were selected with the rectangle tool. The mean pixel intensity readings (total intensity readings/area) for the selected PM regions were recorded and the average values were calculated subsequently. The data shown in Figures 1, 3, 4 were averages from the different images captured at least from 30 individual roots. The data shown in Figure 2 were averages from the different images captured at least from 10 individual roots. The experiments were repeated three times with similar results.

For the measurement of the fluorescence levels at the BFA bodies, the regions of the visible BFA bodies in a single cell of the captured images (over 20 cells for Figure 1; 5-10 cells for Figures 2-4) were selected with the oval tool of Image J. The mean pixel intensity readings (total intensity readings/area) for the selected regions in a single cell were recorded and the mean value of the fluorescence intensity of the BFA bodies in a single cell was calculated by dividing the number of BFA bodies in the single cell (the sum of mean values/the number of BFA bodies). The average fluorescence intensity of the BFA bodies in a captured image were calculated subsequently by dividing the number of cells in the captured image (the sum of average fluorescence intensity of the BFA bodies in a single cell/the number of cells in a captured image). The data shown in Figures 1, 3, 4 were averages from different images captured from at least 30 individual roots. The data shown in Figure 2 were averages from the different images captured at least from 10 individual roots. The experiments were repeated three times with similar results.

For calculation of the fluorescence intensity ratio of BFA/PM, the average fluorescence intensity of the BFA bodies measured in a captured image was divided by the average fluorescence intensity at the PM of the same image. The data shown in Figures 1, 3, 4 were mean values of the images captured from at least 30 individual roots. The data shown in Figure 2 were the mean values from the images captured at least from 10 individual roots. The experiments were repeated three times with similar results.

The BFA-induced internalization of PM-localized proteins was presented as the average number of fluorescence-labeled BFA bodies per cell (Robert et al., 2010). For all the quantitative data, a Student's $t$ test (paired with two-tailed distribution) was used in statistical analysis.

\section{Root Elongation Assay}

The 2-day-old Col-0 seedlings grown on the 1/2 MS agar plates were transferred onto the 1/2 MS agar plates containing different concentration of GSNO for additional 3-4 days. The root length was measured under dissecting microscopy using Image J (http:// rsb.info.nih.gov/ij).

\section{AUTHOR CONTRIBUTIONS}

J-ZL and JP designed the experiments. MN, LZ, Y-FS, CW and YL performed the experiments. J-ZL and JW wrote the manuscript.

\section{FUNDING}

This work was supported by the National Natural Science Foundation of China (31371401 and 31571423 to J-ZL; and 31370313 to JP), and Xin Miao Talent Program of Zhejiang Province (2016R404009).

\section{ACKNOWLEDGMENTS}

The authors thank Dr. Gary Loake for providing the gsnor1-3 mutant. We also thank Mr. Zhen-Chao Li for his technical help in preparation of the figures. 


\section{REFERENCES}

Astier, J., Kulik, A., Koen, E., Besson-Bard, A., Bourque, S., Jeandroz, S., et al. (2012). Protein S-nitrosylation: what's going on in plants? Free Radic. Biol. Med. 53, 1101-1110. doi: 10.1016/j.freeradbiomed.2012.06.032

Beck, M., Zhou, J., Faulkner, C., MacLean, D., and Robatzek, S. (2012). Spatio-temporal cellular dynamics of the Arabidopsis flagellin receptor reveal activation status-dependent endosomal sorting. Plant Cell 24, 4205-4219. doi: 10.1105/tpc.112.100263

Belenghi, B., Romero-Puertas, M. C., Vercammen, D., Brackenier, A., Inzé, D., Delledonne, M., et al. (2007). Metacaspase activity of Arabidopsis thaliana is regulated by S-nitrosylation of a critical cysteine residue. J. Biol. Chem. 282, 1352-1358. doi: 10.1074/jbc.M608931200

Benhar, M., Forrester, M. T., and Stamler, J. S. (2009). Protein denitrosylation: enzymatic mechanisms and cellular functions. Nat. Rev. Mol. Cell Biol. 10, 721-732. doi: $10.1038 / \mathrm{nrm} 2764$

Blilou, I., Xu, J., Wildwater, M., Willemsen, V., Paponov, I., Friml, J., et al. (2005). The PIN auxin efflux facilitator network controls growth and patterning in Arabidopsis roots. Nature 433, 39-44. doi: 10.1038/nature03184

Chen, R., Hilson, P., Sedbrook, J., Rosen, E., Caspar, T., and Masson, P. H. (1998). The Arabidopsis thaliana AGRAVITROPIC 1 gene encodes a component of the polar-auxin-transport efflux carrier. Proc. Natl. Acad.Sci. U.S.A. 95, 15112-15117. doi: 10.1073/pnas.95.25.15112

Chen, R., Sun, S., Wang, C., Li, Y., Liang, Y., An, F., et al. (2009). The Arabidopsis PARAQUAT RESISTANT2 gene encodes an S-nitrosoglutathione reductase that is a key regulator of cell death. Cell Res. 19, 1377-1387. doi: $10.1038 /$ cr.2009.117

Chen, X., Irani, N. G., and Friml, J. (2011). Clathrin-mediated endocytosis: the gateway into plant cells. Curr. Opin. Plant Biol. 14, 674-682. doi: 10.1016/j.pbi.2011.08.006

Du, Y., Tejos, R., Beck, M., Himschoot, E., Li, H., Robatzek, S., et al. (2013). Salicylic acid interferes with clathrin-mediated endocytic protein trafficking. Proc. Natl. Acad. Sci. U.S.A. 110, 7946-7951. doi: 10.1073/pnas.1220205110

Durner, J., Wendehenne, D., and Klessig, D. F. (1998). Defense gene induction in tobacco by nitric oxide, cyclic GMP, and cyclic ADPribose. Proc. Natl. Acad. Sci. U.S.A. 95, 10328-10333. doi: 10.1073/pnas.95.17.10328

Fan, Q. J., and Liu, J. H. (2012). Nitric oxide is involved in dehydration/drought tolerance in Poncirus trifoliata seedlings through regulationof antioxidant systems and stomatal response. Plant Cell Rep. 31, 145-154. doi: 10.1007/s00299-011-1148-1

Feechan, A., Kwon, E., Yun, B. W., Wang, Y., Pallas, J. A., and Loake, G. J. (2005). A central role for S-nitrosothiols in plant disease resistance. Proc. Natl. Acad. Sci. U.S.A. 102, 8054-8059. doi: 10.1073/pnas.0501456102

Feng, J., Wang, C., Chen, Q., Chen, H., Ren, B., Li, X., et al. (2013). S-Nitrosylation of phosphotransfer proteins represses cytokinin signaling. Nat. Commun. 4:1529. doi: $10.1038 /$ ncomms 2541

Forrester, M. T., Thompson, J. W., Foster, M. W., Nogueira, L., Moseley, M. A., and Stamler, J. S. (2009). Proteomic analysis of Snitrosylation and denitrosylation by resin-assisted capture. Nat. Biotechnol. 27, 557-559. doi: 10.1038/nbt.1545

Friml, J., Yang, X., Michniewicz, M., Weijers, D., Quint, A., Tietz, O., et al. (2004). A PINOID-dependent binary switch in apical-basal PIN polar targeting directs auxin efflux. Science 306, 862-865. doi: 10.1126/science.1100618

Gälweiler, L., Guan, C., Müller, A., Wisman, E., Mendgen, K., Yephremov, A., et al. (1998). Regulation of polar auxin transport by AtPIN1 in Arabidopsis vascular tissue. Science 282, 2226-2230. doi: 10.1126/science.282.5397.2226

Gao, Y., Zhang, Y., Zhang, D., Dai, X., Estelle, M., and Zhao, Y. (2015). Auxin binding protein 1 (ABP1) is not required for either auxin signaling or Arabidopsis development. Proc. Natl. Acad. Sci. U.S.A. 112, 2275-2280. doi: 10.1073/pnas.1500365112

Geldner, N., Friml, J., Stierhof, Y. D., Jürgens, G., and Palme, K. (2001). Auxin transport inhibitors block PIN1 cycling and vesicle trafficking. Nature 413, 425-428. doi: 10.1038/35096571

He, Y., Tang, R. H., Hao, Y., Stevens, R. D., Cook, C. W., Ahn, S. M., et al. (2004). Nitric oxide represses the Arabidopsis floral transition. Science 305, 1968-1971. doi: $10.1126 /$ science. 1098837

He, H., Zhan, J., He, L., and Gu, M. (2012). Nitric oxide signaling in aluminum stress in plants. Protoplasma 249, 483-492. doi: 10.1007/s00709-011-0310-5
Hess, D. T., and Stamler, J. S. (2012). Regulation by S-nitrosylation of protein post-translational modification. J. Biol. Chem. 287, 4411-4418. doi: $10.1074 /$ jbc.R111.285742

Hess, D. T., Matsumoto, A., Kim, S. O., Marshall, H. E., and Stamler, J. S. (2005). Protein S-nitrosylation: purview and parameters. Nat. Rev. Mol. Cell Biol. 6, 150-166. doi: 10.1038/nrm1569

Hu, J., Yang, H., Mu, J., Lu, T., Peng, J., Deng, X., et al. (2017). Nitric oxide regulates protein methylation during stress responses in plants. Mol. Cell 67, 702-710. doi: 10.1016/j.molcel.2017.06.031

Klessig, D. F., Durner, J., Noad, R., Navarre, D. A., Wendehenne, D., Kumar, D., et al. (2000). Nitric oxide and salicylic acid signaling in plant defense. Proc. Natl. Acad. Sci. U.S.A. 97, 8849-8855. doi: 10.1073/pnas.97.16.8849

Lamattina, L., García-Mata, C., Graziano, M., and Pagnussa, G. (2003). Nitric oxide: the versatility of an extensive signal molecule. Annu. Rev. Plant Biol. 54, 109-136. doi: 10.1146/annurev.arplant.54.031902.134752

Lee, U., Wie, C., Fernandez, B. O., Feelisch, M., and Vierling, E. (2008). Modulation of nitrosative stress by S-nitrosoglutathione reductase is critical for thermotolerance and plant growth in Arabidopsis. Plant Cell 20, 786-802. doi: 10.1105/tpc.107.052647

Leitner, J., Petrášek, J., Tomanov, K., Retzer, K., Parezova, M., Korbei, B., et al. (2012). Lysine63-linked ubiquitylation of PIN2 auxin carrier protein governs hormonally controlled adaptation of Arabidopsis root growth. Proc. Natl. Acad. Sci. U.S.A. 109, 8322-8327. doi: 10.1073/pnas. 1200824109

Leyser, O. (2006). Dynamic integration of auxin transport and signalling. Curr. Biol. 16, R424-R433. doi: 10.1016/j.cub.2006.05.014

Lin, A., Wang, Y., Tang, J., Xue, P., Li, C., Liu, L., et al. (2012). Nitric oxide and protein Snitrosylation are integral to hydrogen peroxide-induced leaf cell death in rice. Plant Physiol. 158, 451-464. doi: 10.1104/pp.111.184531

Lindermayr, C., Saalbach, G., and Durner, J. (2005). Proteomic identification of S-nitrosylated proteins in Arabidopsis. Plant Physiol. 137, 921-930. doi: 10.1104/pp.104.058719

Lindermayr, C., Saalbach, G., Bahnweg, G., and Durner, J. (2006). Differential inhibition of Arabidopsis methionine adenosyltransferases by protein Snitrosylation. J. Biol. Chem. 281, 4285-4291. doi: 10.1074/jbc.M511635200

Lindermayr, C., Sell, S., Müller, B., Leister, D., and Durner, J. (2010). Redox regulation of the NPR1-TGA1 system of Arabidopsis thaliana by nitric oxide. Plant Cell 22, 2894-2907. doi: 10.1105/tpc.109.066464

Liu, C. (2015). Auxin Binding Protein 1 (ABP1): a matter of fact. J. Integr. Plant Biol. 57, 234-235. doi: 10.1111/jipb.12339

Liu, J. Z., Duan, J., Ni, M., Liu, Z., Qiu, W. L., Whitham, S. A., et al. (2017). Snitrosylation inhibits the kinase activity of tomato phosphoinositide-dependent kinase 1 (PDK1). J. Biol. Chem. doi: 10.1074/jbc.M117.803882. [Epub ahead of print].

Liu, L., Hausladen, A., Zeng, M., Que, L., Heitman, J., and Stamler, J. S. (2001). A metabolic enzyme for S-nitrosothiol conserved from bacteria to humans. Nature 410, 490-494. doi: 10.1038/35068596

Liu, L., Yan, Y., Zeng, M., Zhang, J., Hanes, M. A., Ahearn, G., et al. (2004). Essential roles of S-nitrosothiols in vascular homeostasis and endotoxic shock. Cell 116, 617-628. doi: 10.1016/S0092-8674(04)00131-X

Luschnig, G., and Vert, G. (2014). The dynamics of plant plasma membrane proteins: PINs and beyond. Development 141, 2924-2938. doi: $10.1242 /$ dev.103424

McMahon, H. T., and Boucrot, E. (2011). Molecular mechanism and physiological functions of clathrin-mediated endocytosis. Nat. Rev. Mol. Cell Biol. 12, 517-533. doi: $10.1038 / \mathrm{nrm} 3151$

Muller, A., Guan, C., Gälweiler, L., Tänzler, P., Huijser, P., Marchant, A., et al. (1998). AtPIN2 defines a locus of Arabidopsis for root gravitropism control. EMBO J. 17, 6903-6911. doi: 10.1093/emboj/17.23.6903

Mur, L. A., Mandon, J., Persijn, S., Cristescu, S. M., Moshkov, I. E., Novikova, G. V., et al. (2013). Nitric oxide in plants: an assessment of the current state of knowledge. AoB Plants 5:pls052. doi: 10.1093/aobpla/pls052

Neill, S. J., Desikan, R., Clarke, A., and Hancock, J. T. (2002). Nitric oxide is a novel component of abscisic acid signaling in stomatal guard cells. Plant Physiol. 128, 13-16. doi: 10.1104/pp.010707

Paciorek, T., Zazímalová, E., Ruthardt, N., Petrásek, J., Stierhof, Y. D., KleineVehn, J., et al. (2005). Auxin inhibits endocytosis and promotes its own efflux from cells. Nature 435, 1251-1256. doi: 10.1038/nature03633 
Pan, J., Fujioka, S., Peng, J., Chen, J., Li, G., and Chen, R. (2009). The E3 ubiquitin ligase SCFTIR1/AFB and membrane sterols play key roles in auxin regulation of endocytosis, recycling, and plasma membrane accumulation of the auxin efflux transporter PIN2 in Arabidopsis thaliana. Plant Cell 21, 568-580. doi: 10.1105/tpc.108.061465

Petrásek, J., and Friml, J. (2009). Auxin transport routes in plant development. Development 136, 2675-2688. doi: 10.1242/dev.030353

Renganathan, M., Cummins, T. R., and Waxman, S. G. (2002). Nitric oxide blocks fast, slow, and persistent $\mathrm{Na}^{+}$channels in C-type DRG neurons by S-nitrosylation. J. Neurophysiol. 87, 761-775. doi: 10.1152/jn.00369.2001

Robatzek, S., Chinchilla, D., and Boller, T. (2006). Ligand-induced endocytosis of the pattern recognition receptor FLS2 in Arabidopsis. Genes Dev. 20, 537-542. doi: $10.1101 / \operatorname{gad} .366506$

Robert, S., Kleine-Vehn, J., Barbez, E., Sauer, M., Paciorek, T., Baster, P., et al. (2010). ABP1 mediates auxin inhibition of clathrin-dependent endocytosis in Arabidopsis. Cell 143, 111-121. doi: 10.1016/j.cell.2010.09.027

Romero-Puertas, M. C., Laxa, M., Mattè, A., Zaninotto, F., Finkemeier, I., Jones, A. M., et al. (2007). S-nitrosylation of peroxiredoxin II E promotes peroxynitrite-mediated tyrosine nitration. Plant Cell 19, 4120-4130. doi: 10.1105/tpc.107.055061

Sauer, M., Paciorek, T., Benková, E., and Friml, J. (2006). Immunocytochemical techniques for whole-mount in situ protein localization in plants. Nat. Protoc. 1, 98-103. doi: 10.1038/nprot.2006.15

Sengupta, R., and Holmgren, A. (2012). Thioredoxin and thioredoxin reductase in relation to reversible S-nitrosylation. Antioxid. Redox Signal. 18, 259-269. doi: 10.1089/ars.2012.4716

Serpa, V., Vernal, J., Lamattina, L., Grotewold, E., Cassia, R., and Terenzi, H. (2007). Inhibition of AtMYB2 DNA-binding by nitric oxide involves cysteine S-nitrosylation. Biochem. Biophys. Res. Commun. 361, 1048-1053. doi: 10.1016/j.bbrc.2007.07.133

Shi, Y. F., Wang, D. L., Wang, C., Culler, A. H., Kreiser, M. A., Suresh, J., et al. (2015). Loss of GSNOR1 function leads to compromised auxin signaling and polar auxin transport. Mol. Plant 8, 1350-1365. doi: 10.1016/j.molp.2015.04.008

Shinohara, N., Taylor, C., and Leyser, O. (2013). Strigolactone can promote or inhibit shoot branching by triggering rapid depletion of the auxin efflux protein PIN1 from the plasma membrane. PLoS Biol. 11:e1001474. doi: 10.1371/journal.pbio.1001474

Sokolovski, S., and Blatt, M. R. (2004). Nitric oxide block of outwardrectifying $\mathrm{K}^{+}$ channels indicates direct control by protein nitrosylation in guard cells. Plant Physiol. 136, 4275-4284. doi: 10.1104/pp.104.050344

Spallek, T., Beck, M., Ben Khaled, S., Salomon, S., Bourdais, G., et al. (2013). ESCRT-I Mediates FLS2 endosomal sorting and plant immunity. PLoS Genet. 9:e1004035. doi: 10.1371/journal.pgen.1004035

Stamler, J. S., Simon, D. I., Osborne, J. A., Mullins, M. E., Jaraki, O., Michel, T., et al. (1992). S-nitrosylation of proteins with nitric oxide: synthesis and characterization of biologically active compounds. Proc. Natl. Acad. Sci. U.S.A. 89, 444-448. doi: 10.1073/pnas.89.1.444

Tada, Y., Spoel, S. H., Pajerowska-Mukhtar, K., Mou, Z., Song, J., Wang, C., et al. (2008). Plant immunity requires conformational changes [corrected] of NPR1 via S-nitrosylation and thioredoxins. Science 321, 952-956. doi: 10.1126/science.1156970

Teale, W. D., Paponov, I. A., and Palme, K. (2006). Auxin in action: signalling, transport and the control of plant growth and development. Nat. Rev. Mol. Cell Biol. 7, 847-859. doi: 10.1038/nrm2020

Wang, C., Yan, X., Chen, Q., Jiang, N., Fu, W., Ma, B., et al. (2013). Clathrin light chains regulate clathrin-mediated trafficking, auxin signaling,and development in Arabidopsis. Plant Cell 25, 499-516. doi: 10.1105/tpc.112. 108373

Wang, P., Du, Y., Hou, Y. J., Zhao, Y., Hsu, C. C., Yuan, F., et al. (2015). Nitric oxide negatively regulates abscisic acid signaling in guard cells by $\mathrm{S}$-nitrosylation of OST1. Proc. Natl. Acad. Sci. U.S.A. 112, 613-618. doi: 10.1073/pnas.1423481112

Wang, Y. Q., Feechan, A., Yun, B. W., Shafiei, R., Hofmann, A., Taylor, P., et al. (2009). S-Nitrosylation of AtSABP3 antagonizes the expression of plant immunity. J. Biol. Chem. 284, 2131-2137. doi: 10.1074/jbc.M806782200

Wendehenne, D., Durner, J., Klessig, D. F. (2004). Nitric oxide: a new player in plant signalling and defence responses. Curr. Opin. Plant Biol. 7, 449-455.

Wendehenne, D., Gao, Q. M., Kachroo, A., and Kachroo, P. (2014). Free radicalmediated systemic immunity in plants. Curr. Opin. Plant Biol. 20C, 127-134. doi: 10.1016/j.pbi.2014.05.012

Wisniewska, J., Xu, J., Seifertová, D., Brewer, P. B., Ruzicka, K., Blilou, I., et al. (2006). Polar PIN localization directs auxin flow in plants. Science 312:883. doi: $10.1126 /$ science. 1121356

Woodward, A. W., and Bartel, B. (2005). Auxin: regulation, action, and interaction. Ann. Bot. 95, 707-735. doi: 10.1093/aob/mci083

$\mathrm{Xu}, \mathrm{J}$., and Scheres, B. (2005). Dissection of Arabidopsis ADPRIBOSYLATION FACTOR 1 function in epidermal cell polarity. Plant Cell 17, 525-536. doi: 10.1105/tpc.104.028449

Xu, L., Eu, J. P., Meissner, G., and Stamler, J. S. (1998). Activation of the cardiac calcium release channel (ryanodine receptor) by poly-Snitrosylation. Science 279, 234-237. doi: 10.1126/science.279.5348.234

Xuan, Y., Zhou, S., Wang, L., Cheng, Y., and Zhao, L. (2010). Nitric oxide functions as a signal and acts upstream of AtCaM3 in thermotolerance in Arabidopsis seedlings. Plant Physiol. 153, 1895-1906. doi: 10.1104/pp.110.160424

Yang, H., Mu, J., Chen, L., Feng, J., Hu, J., Li, L., et al. (2015). S-nitrosylation positively regulates ascorbate peroxidase activity during plant stress responses. Plant Physiol. 167, 1604-1615. doi: 10.1104/pp.114.255216

Ye, Y., Li, Z., and Xing, D. (2012). Sorting out the role of nitric oxide in cadmiuminduced Arabidopsis thaliana programmed cell death. Plant Signal. Behav. 7, 1493-1494. doi: 10.4161/psb.21893

Yin, X. J., Volk, S., Ljung, K., Mehlmer, N., Dolezal, K., Ditengou, F., et al. (2007). Ubiquitin lysine 63 chain forming ligases regulate apical dominance in Arabidopsis. Plant Cell 19, 1898-1911. doi: 10.1105/tpc.107.052035

Yun, B. W., Feechan, A., Yin, M., Saidi, N. B., Le Bihan, T., Yu, M., et al. (2011). SNitrosylation of NADPH oxidase regulates cell death in plant immunity. Nature 478, 264-268. doi: 10.1038/nature10427

Zeidler, D., Zähringer, U., Gerber, I., Dubery, I., Hartung, T., Bors, W., et al. (2004). Innate immunity in Arabidopsis thaliana: lipopolysaccharides activate nitric oxide synthase (NOS). Proc. Natl. Acad. Sci. U.S.A. 101, 15811-15816. doi: 10.1073/pnas.0404536101

Conflict of Interest Statement: The authors declare that the research was conducted in the absence of any commercial or financial relationships that could be construed as a potential conflict of interest.

The handling Editor declared a shared affiliation, though no other collaboration, with several of the authors CW and JP.

Copyright (c) $2017 \mathrm{Ni}$, Zhang, Shi, Wang, Lu, Pan and Liu. This is an open-access article distributed under the terms of the Creative Commons Attribution License (CC $B Y)$. The use, distribution or reproduction in other forums is permitted, provided the original author(s) or licensor are credited and that the original publication in this journal is cited, in accordance with accepted academic practice. No use, distribution or reproduction is permitted which does not comply with these terms. 